\title{
Nonlinear centrifugal instabilities in curved free shear layers
}

\author{
Omar Es-Sahli*1, Adrian Sescu ${ }^{\dagger 1}$, and Mohammed Afsar ${ }^{\ddagger 2}$ \\ ${ }^{1}$ Department of Aerospace Engineering, Mississippi State University, MS 39762 \\ ${ }^{2}$ Department of Mechanical \& Aerospace Engineering, Strathclyde University, 75 Montrose \\ St. Glasgow, G1 1XJ, UK
}

\begin{abstract}
Curved free shear layers exist in many engineering problems involving complex flow geometries, such as the backward facing step flow, flows with wall injection, the flow inside side-dump combustors, or flows generated by vertical axis wind turbines, among others. Most of the studies involving centrifugal instabilities have been focused on wall flows where Taylor instabilities between two rotating concentric cylinders or Görtler vortices in boundary layers resulting from the imbalance between centrifugal effects and radial pressure gradients, are generated. Curved free shear layers, however, did not receive sufficient attention. An examination of the stability characteristics and the flow structures associated with curved free shear flows should provide a better understanding of these complex flow problems. In this work, we study the development of Görtler vortices inside a curved shear layer in both the incompressible and compressible regimes using a numerical solution to a parabolized form of the Navier-Stokes equations, in the assumption that the streamwise wavenumber associated with the vortex flow is much smaller than the crossstream wavenumbers. Various results consisting of contour plots of centrifugal instabilites in crossflow planes, and energy and streak amplitude distributions along the streamwise direction are reported and discussed. In addition, we conduct a biglobal stability analysis to study the growth rates and the eigenmodes associated with these flows.
\end{abstract}

\section{Introduction}

The stability of an incompressible flow in curved shear layers depend on the velocity difference across the shear layer, and the streamline curvature. For a free shear layer with no curvature (as known as a plane shear layer) the Kelvin-Helmholtz instability is the dominant mechanism. ${ }^{17}$ In this case, two-dimensional disturbances are more unstable than their three-dimensional counterpart, ${ }^{27}$ and the Kelvin-Helmholtz instability introduces predominantly spanwise oriented vortices. Rayleigh ${ }^{21}$ proved that the existence of an inflection point in the basic velocity profile was a necessary condition for the Kelvin-Helmholtz instability. For the case of a flow in curved mixing layer with an inflectional velocity profile, it may be expected that the Kelvin-Helmholtz instability mechanism is still present, but it will be mixed with centrifugal instabilities in the form of streamwise oriented Görtler type vortices.

Görtler vortices are mostly known to appear inside a boundary layer flow along a concave surface due to the imbalance between radial pressure gradients and centrifugal forces (e.g., Gortler, ${ }^{7}$ Hall, ${ }^{8-10}$ Swearingen \& Blackwelder, ${ }^{28}$ Malik \& Hussaini, ${ }^{16}$ Saric ${ }^{23}$ Li \& Malik, ${ }^{12}$ Boiko et al., ${ }^{2}$ Wu et al., ${ }^{31}$ or Sescu et al.,${ }^{25,26}$ Ren \& Fu, ${ }^{22}$ Dempsey et al., ${ }^{4} \mathrm{Xu}$ et al. ${ }^{32}$ ). For highly curved walls, for example, vortex formation occurs more rapidly and can significantly alter the mean flow causing the laminar flow to breakdown into turbulence. Under certain conditions, Görtler vortices can be efficient precursors to transition: they consist of counter-rotating streamwise vortices that grow at a certain rate, depending on the surface curvature and the receptivity of the boundary layer to environmental disturbances and surface imperfections. It is important

*PhD Student, Department of Aerospace Engineering, Mississippi State University; AIAA Student Member.

${ }^{\dagger}$ Associate Professor, Department of Aerospace Engineering, Mississippi State University; Associate Fellow AIAA.

${ }^{\ddagger}$ Chancellor Fellow, Department of Mechanical \& Aerospace Engineering; AIAA member. 
to understand the fundamental processes that lie behind these centrifugal instabilities, and to predict their occurrence using efficient and tractable methods.

The first to study this problem was Görtler ${ }^{7}$ himself (the instabilities actually bear his name), who obtained solutions of the disturbance equations in the form of streamwise, counter-rotating vortices. Although in reality the flow over concave surfaces is not parallel, Görtler made a local parallel flow assumption, and employed normal mode analysis to show that the instabilities may occur when a certain dimensionless parameter, $R e \sqrt{\delta / R}$, where $R e$ is Reynolds number in terms of free-stream velocity, the boundary layer thickness is $\delta$, and $R$ is the radius of the curvature, exceeds a critical value. Gregory and Walker ${ }^{5}$ performed the first set of experimental measurements using china-clay surface visualization to educe Görtler vortex structure, while Tani ${ }^{30}$ later on used hot-wire measurements of the flow inside the boundary layer for the detection method. These experiments, and others that followed, showed that the type of instability is convective, and develops along the streamwise direction.

Other early studies relied on so-called normal-mode analysis as the approach of reducing the governing Navier-Stokes equations to a set of ordinary differential equations (assuming a constant growth rate along the streamwise direction), the Görtler problem is then solved using an eigenvalue analysis. Hall ${ }^{9}$ was the first to consider a nonparallel formulation of the Görtler problem, wherein the solution to the resulting partial differential equations was obtained by a marching technique. He found that the stability depends on the location and form of the initial disturbance and concluded that it was not possible to determine a unique neutral stability curve for the Görtler problem. However, Day et al. ${ }^{3}$ found modest differences between the normal-mode analysis and the marching scheme in their investigations, concluding that the normal-mode analysis can be used for engineering studies via appropriate empirical corrections. Boiko et al. ${ }^{\text {? }}$ studied (theoretically and experimentally) the steady and unsteady linear Görtler instability. They carried out calculations based on locally-parallel and nonlocal/non-parallel linear-stability theories, and compared the results with experimental data.

Centrifugal instabilities on curved incompressible free shear layers has been studied theoretically and numerically. Plesniak et al. ${ }^{19,20}$ conducted extensive experimental measurements in order to study curved two-stream mixing layers to show how centrifugal effects yield streamwise vortices. The untripped case within this suite of experiments exhibited organized streamwise vorticity, while the tripped cases did not. They explained this by the existence of spatially stationary streamwise vortices, which are seen to provide extra entrainment (Bell \& Mehta $^{1}$ showed this for a plane mixing layer). Hu et al. ${ }^{11}$ and Liou, ${ }^{14}$ concerned with the effect of the curvature on the inflectional Rayleigh modes, found that it appears to be small, although the curvature excites an unstable three-dimensional disturbance with the amplitude increasing as the streamwise wavenumber decreases. Otto et al., ${ }^{18}$ through a combined analytical and numerical study, showed that the unstable modes are largely dependent on surface curvature. Otto et al. ${ }^{18}$ also employed numerical simulations to solve for a set of parabolic equations, where the wavenumber and the Görtler number were taken to be of oder one. They found that as the difference between the free-stream speeds increased, the layer became more susceptible to centrifugal instabilities.

The focus of the present work is on the analysis of centrifugal instabilities that develop in curved free shear layers in both incompressible and compressible regimes, by using a numerical algorithm that solves a parabolized version of the Navier-Stokes equations, under the assumption that the streamwise wavenumber associated with the disturbances is much smaller than the crossflow wavenumbers. We targeted the nonlinear development of these developing centrifugal instabilities, which resembles many similarities with the Görtler vortices that evolve in boundary layer flows over concave surfaces. We vary the spanwise separation that characterizes the upstream disturbance (dictating the spanwise separation of the downstream disturbances) and the Mach number of the fastest stream. In addition, we conduct a biglobal stability analysis to study the growth rates and the eigenmodes associated with these flows, which provide information about the types of secondary instability mechanism (varicose or sinuous) that may be prevalent here.

In section II, the mathematical model is introduced and described; we include a discussion of the scalings of appropriate independent and dependent variables, the initial and boundary conditions, as well as the numerical algorithm. In section III, results for various spanwise separations and Mach numbers are shown in addition to results from the biglobal stability analysis. Concluding remarks are given in section IV. 


\section{Problem formulation and numerical algorithm}

\section{A. Scalings}

All dimensional spatial coordinates $\left(x^{*}, y^{*}, z^{*}\right)$ are normalized by the spanwise separation $\lambda^{*}$, while the dependent variables by their respective freestream values, except the pressure, which is normalized by the dynamic pressure:

$$
\begin{gathered}
\bar{t}=\frac{t^{*}}{\lambda^{*} / V_{\infty}^{*}} ; \quad \bar{x}=\frac{x^{*}}{\lambda^{*}} ; \quad \bar{y}=\frac{y^{*}}{\lambda^{*}} ; \quad \bar{z}=\frac{z^{*}}{\lambda^{*}} \\
\bar{u}=\frac{u^{*}}{V_{\infty}^{*}} ; \quad \bar{v}=\frac{v^{*}}{V_{\infty}^{*}} ; \quad \bar{w}=\frac{w^{*}}{V_{\infty}^{*}} ; \quad \bar{\rho}=\frac{\rho^{*}}{\rho_{\infty}^{*}} \\
\bar{p}=\frac{p^{*}-p_{\infty}^{*}}{\rho_{\infty}^{*} V_{\infty}^{* 2}} ; \quad \bar{T}=\frac{T^{*}}{T_{\infty}^{*}} ; \quad \bar{\mu}=\frac{\mu^{*}}{\mu_{\infty}^{*}} ; \quad \bar{k}=\frac{k^{*}}{k_{\infty}^{*}}
\end{gathered}
$$

where $\lambda^{*}$ is the spanwise wavelength of the disturbances, $\left(u^{*}, v^{*}, w^{*}\right)$ are the velocity components, $\rho^{*}$ the density, $p^{*}$ is pressure, $T^{*}$ temperature, $\mu^{*}$ dynamic viscosity, $k^{*}$ thermal conductivity, and all quantities with $\infty$ at the subscript represent conditions at infinity.

Reynolds number based on the spanwise separation, Mach number and Prandtl number are defined as

$$
R_{\lambda}=\frac{\rho_{\infty}^{*} V_{\infty}^{*} \lambda^{*}}{\mu_{\infty}^{*}}, \quad M a=\frac{V_{\infty}^{*}}{a_{\infty}^{*}}, \quad \operatorname{Pr}=\frac{\mu_{\infty}^{*} C_{p}}{k_{\infty}^{*}}
$$

where $\mu_{\infty}^{*}, a_{\infty}^{*}$ and $k_{\infty}^{*}$ are freestream dynamic viscosity, speed of sound and thermal conductivity, respectively, and $C_{p}$ is the specific heat at constant pressure. As for boundary layer flows over curved surfaces, we here define the equivalent global Görtler number as

$$
G_{\lambda}=\frac{R_{\lambda}^{2} \lambda^{*}}{r^{*}}
$$

where $r^{*}$ is the radius of the curvature.

\section{B. Boundary region equations: a parabolized form of the Navier-Stokes equations}

If the streamwise wavenumber of the disturbances evolving inside the shear layer are much larger that the wavenumbers corresponding to the crossflow directions, then the Navier-Stokes equations can be transformed into a parabolic set of equations in the framework of high Reynolds number asymptotics.

For a full compressible flow, the primitive form of the Navier-Stokes equations in non-dimensional variables are considered here in the form

$$
\begin{gathered}
\frac{D \bar{\rho}}{D t}+\rho\left(\frac{\partial \bar{u}}{\partial \bar{x}}+\frac{\partial \bar{v}}{\partial \bar{y}}+\frac{\partial \bar{w}}{\partial \bar{z}}\right)=0 \\
\bar{\rho} \frac{D \bar{u}}{D \bar{t}}=-\frac{\partial \bar{p}}{\partial \bar{x}}+\frac{1}{R e_{\lambda}} \frac{\partial}{\partial \bar{x}}\left[\frac{2}{3} \mu\left(2 \frac{\partial \bar{u}}{\partial \bar{x}}-\frac{\partial \bar{v}}{\partial \bar{y}}-\frac{\partial \bar{w}}{\partial \bar{z}}\right)\right]+\frac{\partial}{\partial \bar{y}}\left[\mu\left(\frac{\partial \bar{u}}{\partial \bar{y}}+\frac{\partial \bar{v}}{\partial \bar{x}}\right)\right]+\frac{\partial}{\partial \bar{z}}\left[\mu\left(\frac{\partial \bar{w}}{\partial \bar{x}}+\frac{\partial \bar{u}}{\partial \bar{z}}\right)\right] \\
\bar{\rho} \frac{D \bar{v}}{D \bar{t}}=-\frac{\partial \bar{p}}{\partial \bar{y}}+\frac{1}{R e_{\lambda}} \frac{\partial}{\partial \bar{y}}\left[\frac{2}{3} \mu\left(2 \frac{\partial \bar{v}}{\partial \bar{y}}-\frac{\partial \bar{u}}{\partial \bar{x}}-\frac{\partial \bar{w}}{\partial \bar{z}}\right)\right]+\frac{\partial}{\partial \bar{x}}\left[\mu\left(\frac{\partial \bar{v}}{\partial \bar{x}}+\frac{\partial \bar{u}}{\partial \bar{y}}\right)\right]+\frac{\partial}{\partial \bar{z}}\left[\mu\left(\frac{\partial \bar{v}}{\partial \bar{z}}+\frac{\partial \bar{w}}{\partial \bar{y}}\right)\right] \\
\bar{\rho} \frac{D \bar{w}}{D \bar{t}}=-\frac{\partial \bar{p}}{\partial \bar{z}}+\frac{1}{R e_{\lambda}} \frac{\partial}{\partial \bar{z}}\left[\frac{2}{3} \mu\left(2 \frac{\partial \bar{w}}{\partial \bar{z}}-\frac{\partial \bar{u}}{\partial \bar{x}}-\frac{\partial \bar{v}}{\partial \bar{y}}\right)\right]+\frac{\partial}{\partial \bar{x}}\left[\mu\left(\frac{\partial \bar{w}}{\partial \bar{x}}+\frac{\partial \bar{u}}{\partial \bar{z}}\right)\right]+\frac{\partial}{\partial \bar{y}}\left[\mu\left(\frac{\partial \bar{v}}{\partial \bar{z}}+\frac{\partial \bar{w}}{\partial \bar{y}}\right)\right]
\end{gathered}
$$




$$
\begin{gathered}
\bar{\rho} \frac{D \bar{T}}{D \bar{t}}=\frac{1}{\operatorname{Pr}_{\operatorname{Re}}}\left[\frac{\partial}{\partial \bar{x}}\left(k \frac{\partial \bar{T}}{\partial \bar{x}}\right)+\frac{\partial}{\partial \bar{y}}\left(k \frac{\partial \bar{T}}{\partial \bar{y}}\right)+\frac{\partial}{\partial \bar{z}}\left(k \frac{\partial \bar{T}}{\partial \bar{z}}\right)\right] \\
-\quad(\gamma-1) M_{\infty}^{2}\left[p\left(\frac{\partial \bar{u}}{\partial \bar{x}}+\frac{\partial \bar{v}}{\partial \bar{y}}+\frac{\partial \bar{w}}{\partial \bar{z}}\right)-\frac{2}{3} \mu\left(\frac{\partial \bar{u}}{\partial \bar{x}}+\frac{\partial \bar{v}}{\partial \bar{y}}+\frac{\partial \bar{w}}{\partial \bar{z}}\right)^{2}\right] \\
+\quad(\gamma-1) M_{\infty}^{2} \frac{\mu}{\operatorname{Re} \lambda}\left[2\left(\frac{\partial \bar{u}}{\partial \bar{x}}\right)^{2}+2\left(\frac{\partial \bar{v}}{\partial \bar{y}}\right)^{2}+2\left(\frac{\partial \bar{w}}{\partial \bar{z}}\right)^{2}+\left(\frac{\partial \bar{u}}{\partial \bar{y}}+\frac{\partial \bar{v}}{\partial \bar{x}}\right)^{2}+\left(\frac{\partial \bar{w}}{\partial \bar{x}}+\frac{\partial \bar{u}}{\partial \bar{z}}\right)^{2}+\left(\frac{\partial \bar{v}}{\partial \bar{z}}+\frac{\partial \bar{w}}{\partial \bar{y}}\right)^{2}\right]
\end{gathered}
$$

where

$$
\frac{D}{D \bar{t}}=\frac{\partial}{\partial \bar{t}}+\bar{u} \frac{\partial}{\partial \bar{x}}+\bar{v} \frac{\partial}{\partial \bar{y}}+\bar{w} \frac{\partial}{\partial \bar{z}}
$$

is the substantial derivative. The pressure $p$, the temperature $T$ and the density of the fluid are combined in the equation of state in non-dimensional form, $\bar{p}=\bar{\rho} \bar{T} / \gamma M_{\infty}^{2}$, assuming that non-chemically-reacting flows are considered. Other notations include the dynamic viscosity $\mu$, Reynolds number $R e=\rho_{\infty} V_{\infty}^{*} \lambda^{*} / \mu$ based on a characteristic velocity $V_{\infty}^{*}$, and a characteristic length $L$, the free-stream Mach number $M_{\infty}=V_{\infty}^{*} / a_{\infty}^{*}$. The dynamic viscosity and thermal conductivity $k$ is linked to the temperature using a power law in dimensionless form,

$$
\mu=T^{b} ; \quad k=\frac{C_{p} \mu}{\operatorname{Pr}}
$$

where $b=0.76, C_{p}=\gamma R /(\gamma-1), \gamma=1.4$, and $\operatorname{Pr}=0.72$ for air.

In the assumption that the streamwise wavenumber of the disturbance is much larger, we assumed that $x / R_{\lambda}=O(1)$, which implies that the scales $x=\bar{x} / R_{\lambda}, t=\bar{t} / R_{\lambda}$ can be considered. Also, the crossflow velocity components and the pressure variation are expected to be small. This suggests the scaling:

$$
u=\bar{u} ; \quad v=\bar{v} / R_{\lambda} ; \quad w=\bar{w} / R_{\lambda} ; \quad \rho=\bar{\rho} ; \quad p=\bar{p} / R_{\lambda}^{2} ; \quad T=\bar{T} ; \quad \mu=\bar{\mu} ; \quad k=\bar{k} ;
$$

Working out the order-of-magnitude analysis of the Navier-Stokes equations, we obtain the parabolic set of equations (which we call compressible boundary region equations)

$$
\begin{aligned}
& \frac{D \rho}{D t}+\rho \nabla \cdot \vec{V}=0 \\
& \rho \frac{D u}{D t}=\nabla_{c} \cdot\left(\mu \nabla_{c} u\right) \\
& \rho \frac{D v}{D t}=-\frac{\partial p}{\partial y}+\frac{\partial}{\partial y}\left[\frac{2}{3} \mu\left(3 \frac{\partial v}{\partial y}-\nabla \cdot \vec{V}\right)\right]+\frac{\partial}{\partial x}\left(\mu \frac{\partial u}{\partial y}\right)+\frac{\partial}{\partial z}\left[\mu\left(\frac{\partial v}{\partial z}+\frac{\partial w}{\partial y}\right)\right]-G_{\lambda} u^{2} \\
& \rho \frac{D w}{D t}=-\frac{\partial p}{\partial z}+\frac{\partial}{\partial z}\left[\frac{2}{3} \mu\left(3 \frac{\partial w}{\partial z}-\nabla \cdot \vec{V}\right)\right]+\frac{\partial}{\partial x}\left(\mu \frac{\partial u}{\partial z}\right)+\frac{\partial}{\partial y}\left[\mu\left(\frac{\partial v}{\partial z}+\frac{\partial w}{\partial y}\right)\right] \\
& \rho \frac{D T}{D t}=\frac{1}{P r} \nabla_{c} \cdot\left(k \nabla_{c} T\right)+(\gamma-1) M_{\infty}^{2} \mu\left[\left(\frac{\partial u}{\partial y}\right)^{2}+\left(\frac{\partial u}{\partial z}\right)^{2}\right]
\end{aligned}
$$

where $\nabla_{c}$ is the crossflow nabla operator:

$$
\nabla_{c}=\frac{\partial}{\partial y} \vec{j}+\frac{\partial}{\partial z} \vec{k}
$$

The mean inflow condition is given as a tangent hyperbolic function:

$$
u=1-\frac{1}{2} \Delta u\left[1-\tanh \left(\alpha\left(y-y_{0}\right)\right)\right]
$$


where $\alpha$ is the width of the shear layer, $y_{0}$ the location of the shear layer, and $\Delta u$ is the velocity difference across the shear layer. Temperature is obtain from the Crocco-Busemann equation:

$$
T=-\frac{1}{2} u^{2}+\left(\frac{1-\frac{u_{2}}{u_{1}}}{\gamma-1}+\frac{M_{\infty}}{2}\right) u+\frac{u_{2}}{u_{1}(\gamma-1)}+\frac{M_{\infty}^{2}}{2}-M_{\infty}\left(\frac{1-\frac{u_{2}}{u_{1}}}{\gamma-1}+\frac{M_{\infty}}{2}\right)
$$

where $u_{1}$ and $u_{2}$ are the velocity in the two regions separated by the shear layer.

A small artificial disturbance is imposed on the base flow in the form:

$$
v^{\prime}=A \cos \left(\frac{\pi z}{\lambda}\right) \exp \left[-\frac{\left(y-y_{0}\right)^{2}}{\sigma^{2}}\right]
$$

where $A$ is a small amplitude, $\lambda$ is the spanwise wavenumber (dictating the spanwise separation of the centrifugal instabilities), and $\sigma$ represents the extent of the disturbance in the $y$-direction.

\section{Biglobal linear stability analysis}

Modal flow linear instability is considered by perturbing the solution to the Navier-Stokes equations to determine whether a small-amplitude disturbance that sets itself into the shear layer has a tendency to either grow or decay. The basic state is assumed independent of one spatial coordinate, $x$, in a Cartesian framework. Flow quantities are then decomposed as:

$$
q(x, y, z, t)=\bar{q}(y, z)+\epsilon \tilde{q}(x, y, z, t)
$$

where $\bar{q}=(\bar{\rho}, \bar{u}, \bar{v}, \bar{w}, \bar{T})$ is the mean crossflow and $\bar{q}=(\tilde{\rho}, \tilde{u}, \tilde{v}, \tilde{w}, \tilde{T})$ is a $3 \mathrm{D}$ disturbance. Unlike the incompressible case, pressure or density is a predictive variable in, rather than a constraint. Substituting this into the Navier-Stokes equations, subtracting the equations from the base flow, and linearizing, the linearized compressible Navier-Stokes equations are obtained. We then consider the ansatz

$$
\tilde{q}(x, y, z, t)=\hat{q}(y, z) \exp [i(\alpha x-\Omega t)],
$$

where $\alpha$ is the streamwise wavenumber, and $\Omega$ is a complex eigenvalue, and plug it into the linerized NavierStokes equations. The real part of the eigenvalue, $\Omega$, is related with the frequency of the global eigenmode, while the imaginary part represents its growth rate.

The eigenvalue problem is obtained in the form

$$
[A]\{Q\}+[B]\{Q\}_{y}+[C]\{Q\}_{z}+[D]\{Q\}_{y y}+[E]\{Q\}_{y z}+[F]\{Q\}_{z z}=\omega[G]\{Q\},
$$

where $y$ at the subscript represents a derivative with respect to $y,\{Q\}=\{p, u, v, w, \tau\}^{T}$ is the vector of eigenmodes, and the coefficients of all matrices are given in the appendix. This equation is subject to appropriate boundary conditions (disturbances are 0 at infinity, for example). This eigenvalue problem is solved to determine the complex frequency for a given wavenumber, where the imaginary part of the frequency represents the growth rate of the disturbance.

\section{Results and Discussion}

Numerical simulations for a curved free shear layer with the Mach number ranging between 0.2 and 4.0 in the faster stream were carried out. The relative velocity difference across the shear layer is 0.2 , while the Reynolds number (per unit length) and the global Görtler number are commensurate with the freestream velocity (the radius of curvature is $2 \mathrm{~m}$ ). The spanwise separation of the vortices is varied between $0.3 \mathrm{~cm}$ and $1.2 \mathrm{~cm}$. The flow domain and the grid are shown in figure 1; stretching was used in the radial direction, towards the farfield boundaries, while equally spaced grid points were use in the streamwise and spanwise directions. In fact, there is no grid in the streamwise direction since the equations are parabolic and the solution advances in a marching procedure, but the spatial step along the streamwise marching direction is constant.

Centrifugal instabilities are excited by a steady small disturbance of non-dimensional amplitude of 0.01 (relative to the fastest stream), and imposed on the base flow at the inflow boundary. No disturbances are imposed to excite Kelvin-Helmholtz type instabilities, as the focus is on the development on the longitudinal centrifugal instabilities in the form of Görtler vortices. 


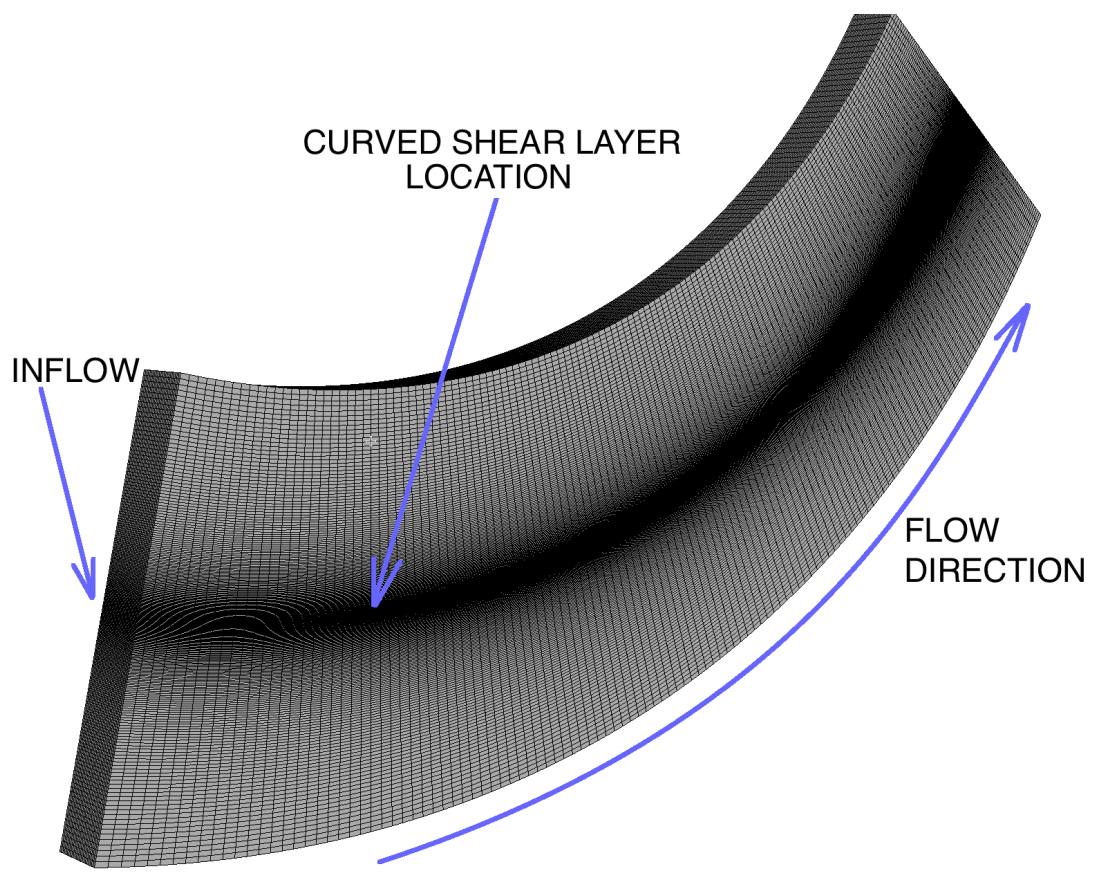

Figure 1. Flow domain and grid configuration.

In figures 2-5, we plot contours of velocity magnitude in several crossflow planes, for different spanwise separations and different Mach numbers, in both incompressible and compressible regimes; the upper stream (shown in dark red) is the faster flow. The results in figure 2 are for the lowest Mach number, 0.2, corresponding to the incompressible regime. As a result, the Reynolds number associated with this flow is smaller than the other cases, which implies a higher diffusion in the mixing. The top row in figure 2 indicates that for the smaller spanwise separation of $0.3 \mathrm{~cm}$ the centrifugal instability is 'weak' in the sense that the mixing between the two streams is not efficient. As the spanwise separation is increased, the mushroom shapes start to show up, as seen in the other three rows of figure 2. In figure 3, corresponding to the Mach number 0.8 , the shapes of the instabilities for the smallest spanwise separation, 0,3 , look slightly different from the incompressible case. The mixing is increased as the spanwise separation is increased, and the large spanwise separations in the last two rows show some spiraling streaks at the third stramwise location, which break down in the next spanwise separations.

In the supersonic regime, plotted in figures 4 and 5 for Mach 2.0 and 4.0, respectively, the mixing is more efficient, and the spiraling streaks are more visible at multiple spanwise separations. We also noticed secondary mushroom shapes developing at some spanwise separations, such as the last two streamwise locations for the spanwise separation of $\lambda=1.2$ (figure 4d). So, we identify the 'primary' mushroom shape the one that develops as the main instability as seen, for example, at the second streamwise locations in figure 4 or 5 . The 'secondary' mushroom shape is defined as the instability that develops from the edge of the main/primary mushroom cap, the best seen in the last streamwise location of figure $4 \mathrm{~d}$. 


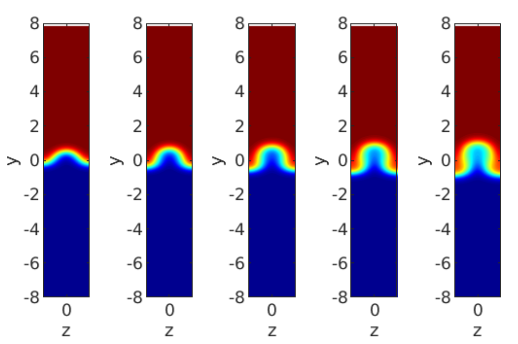

a)
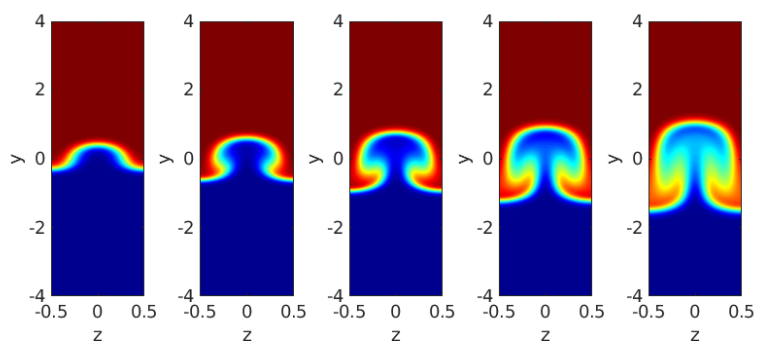

b)
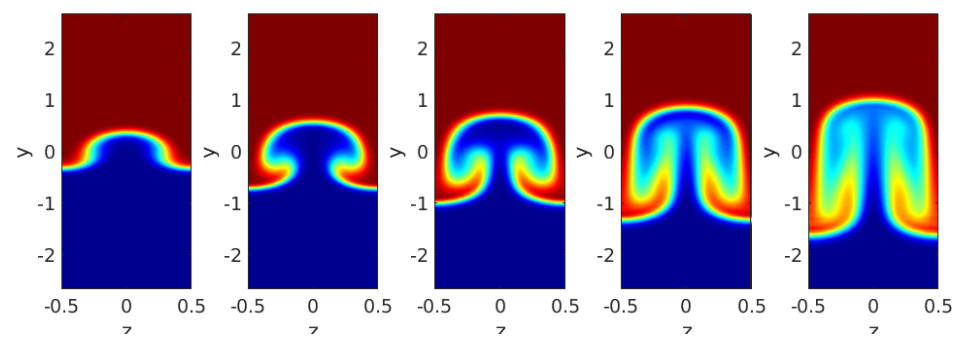

c)
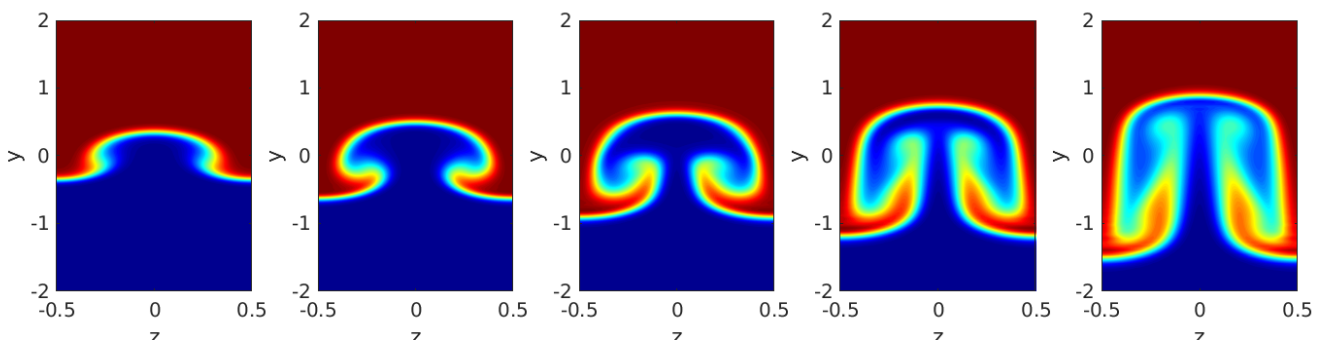

d)

Figure 2. Contour plots of the streamwise velocity at different streamwise locations, for $M=0.2$ : a) $\lambda=0.3$; b) $\lambda=0.6$; b) $\lambda=0.9$; b) $\lambda=1.2$. 


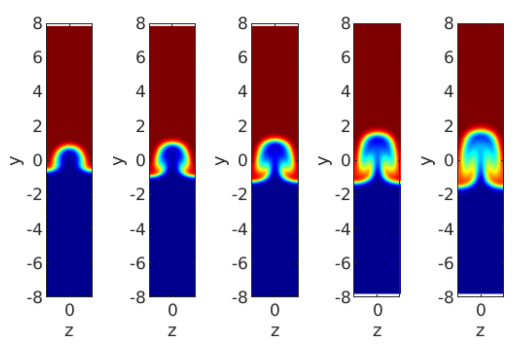

a)
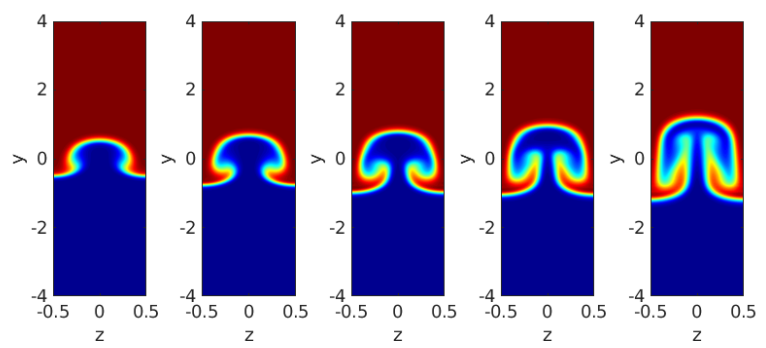

b)
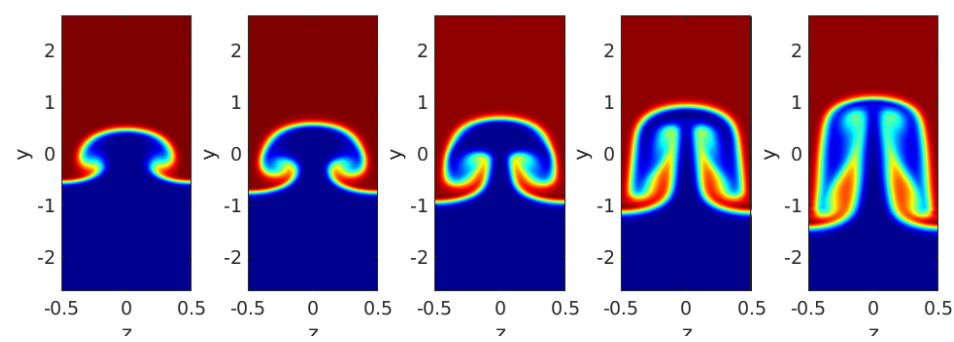

c)
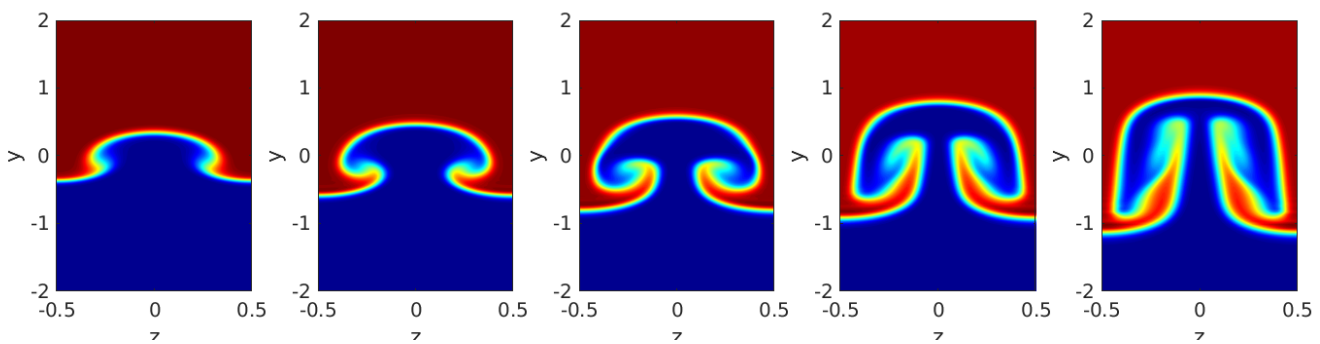

d)

Figure 3. Contour plots of the streamwise velocity at different streamwise locations, for $M=0.8$ : a) $\lambda=0.3$; b) $\lambda=0.6$; b) $\lambda=0.9$; b) $\lambda=1.2$. 


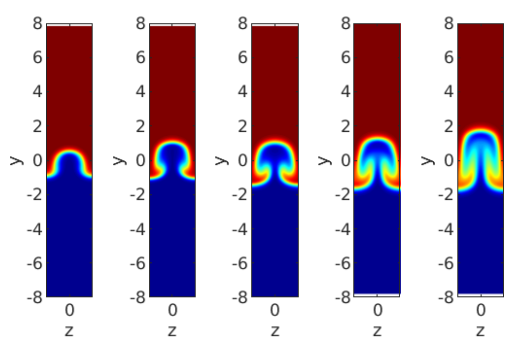

a)
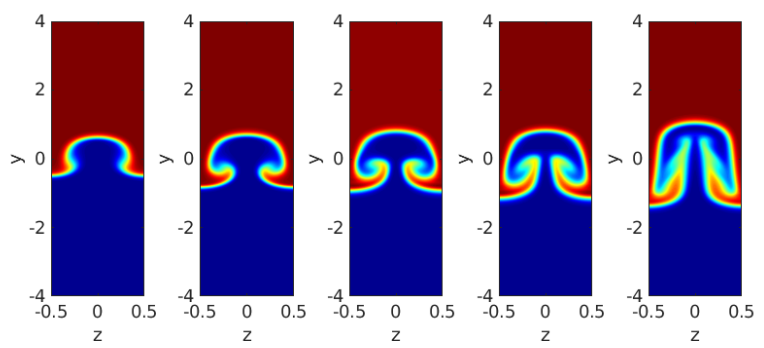

b)
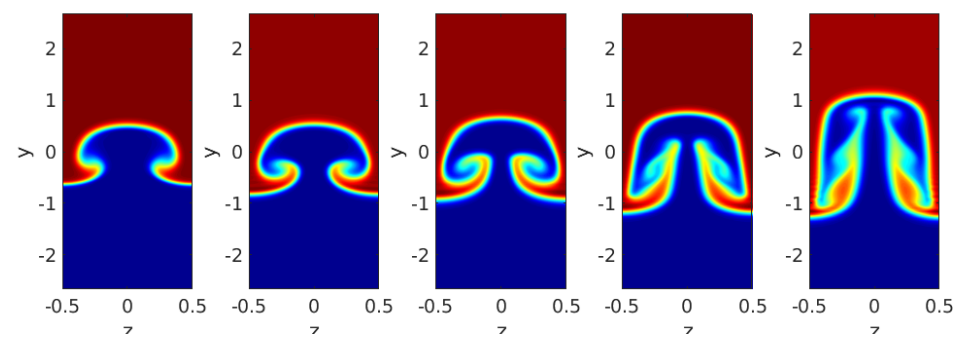

c)
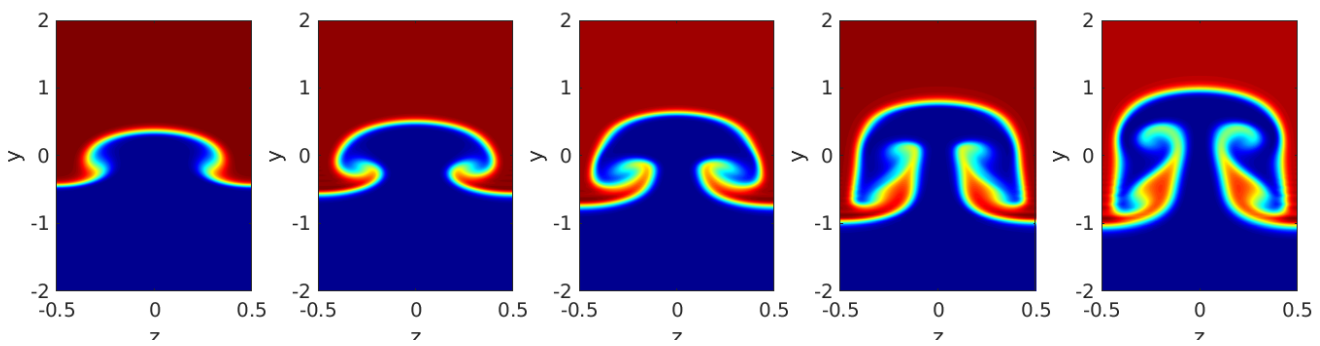

d)

Figure 4. Contour plots of the streamwise velocity at different streamwise locations, for $M=2.0$ : a) $\lambda=0.3$; b) $\lambda=0.6$; b) $\lambda=0.9$; b) $\lambda=1.2$. 


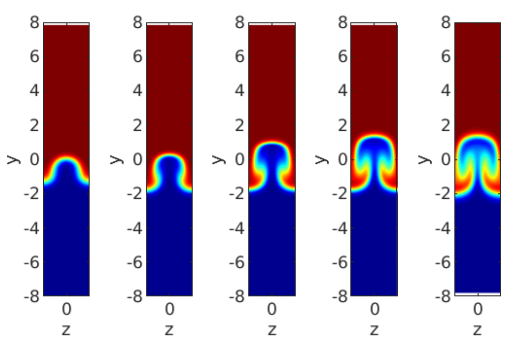

a)
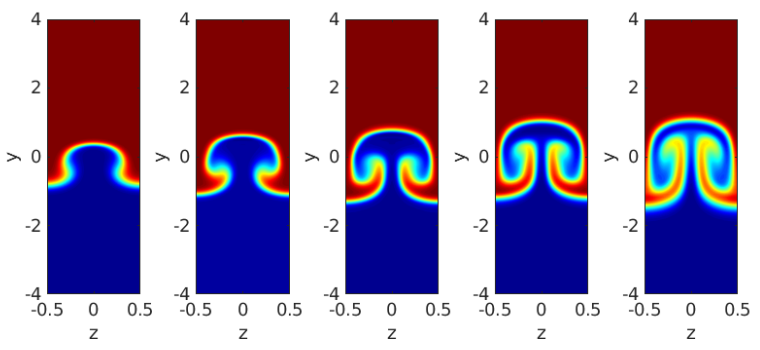

b)
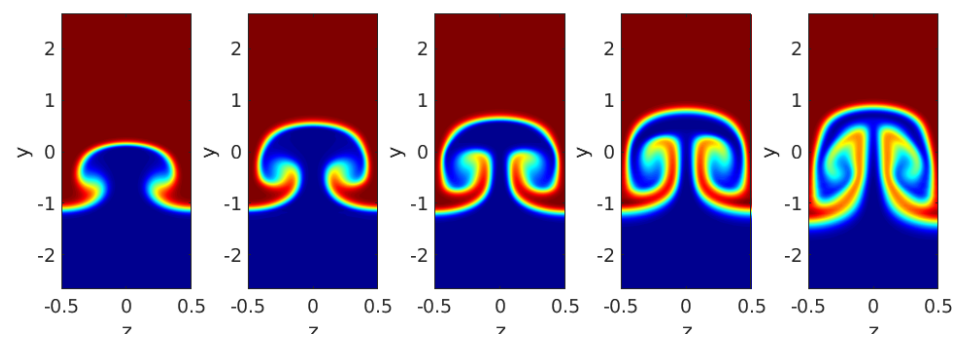

c)
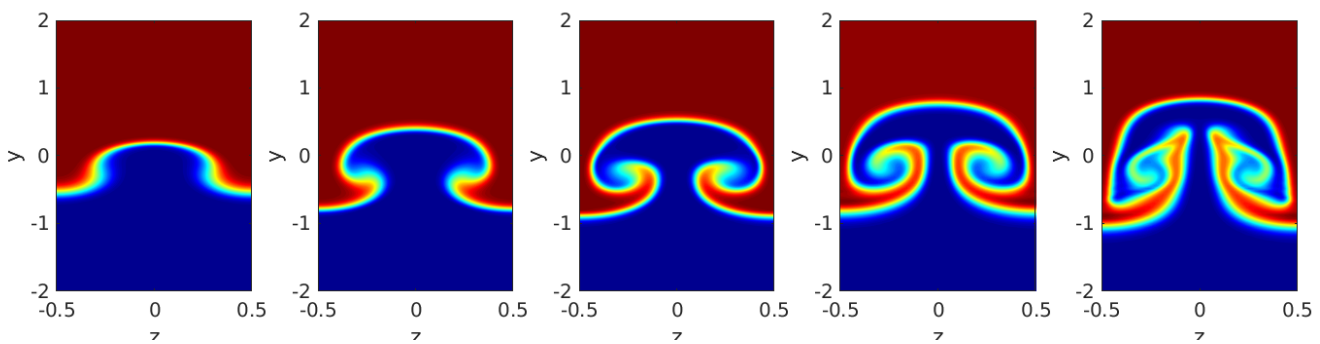

d)

Figure 5. Contour plots of the streamwise velocity at different streamwise locations, for $M=4.0$ : a) $\lambda=0.3$; b) $\lambda=0.6$; b) $\lambda=0.9$; b) $\lambda=1.2$.

Figure 6 shows the kinetic energy distribution of the disturbance, calculated according to

$$
E(x)=\int_{z_{1}}^{z_{2}} \int_{-\infty}^{\infty}\left[\left|u(x, y, z)-u_{m}(x, y)\right|^{2}+\left|v(x, y, z)-v_{m}(x, y)\right|^{2}+\left|w(x, y, z)-w_{m}(x, y)\right|^{2}\right] d z d y
$$

where $u_{m}(x, y), v_{m}(x, y)$, and $w_{m}(x, y)$ are the spanwise mean components of velocity, and $z_{1}$ and $z_{2}$ are the coordinates of the boundaries in the spanwise direction. In figure 6, this energy is plotted (in logarithmic scale on the vertical axis) as a function of the streamwise coordinate, for different spanwise separations and different Mach numbers. As the spanwise separation is increased, the scaled kinetic energy maximum increases. Also, the streamwise location where the energy saturation (the point where the energy starts to level off) occurs, moves downstream as the spanwise separation is increased. 


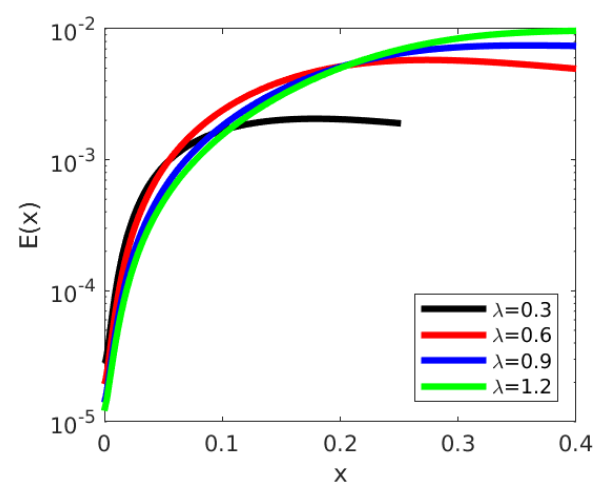

a)

c)

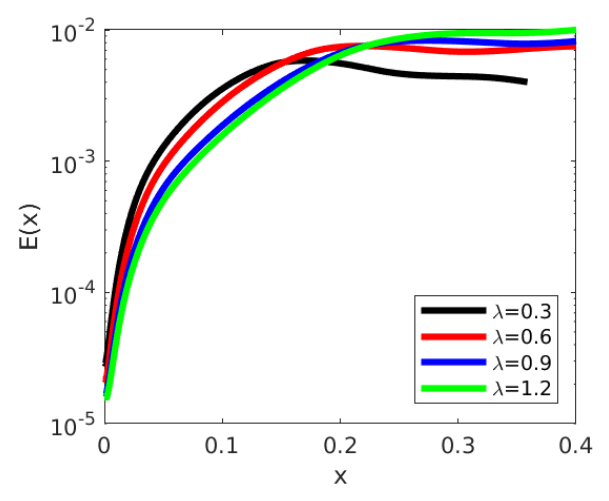

b)

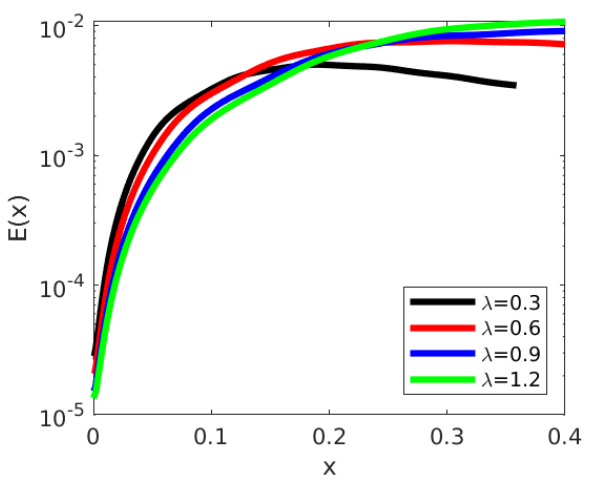

d)

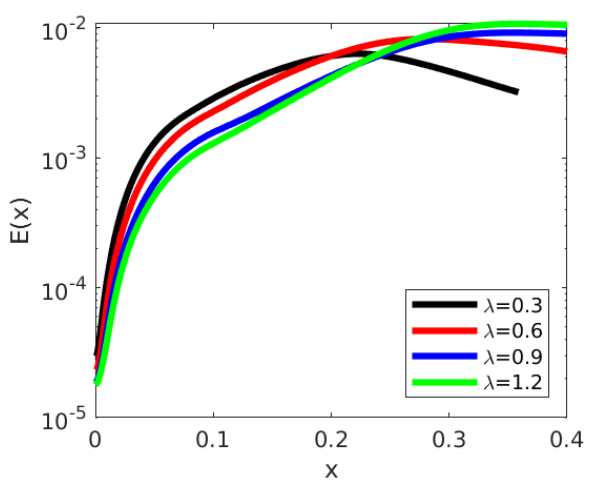

Figure 6. Vortex energy distribution: a) $M=0.2$; b) $M=0.8$; c) $M=2.0$; d) $M=4.0$.

Streak amplitude is defined here as the difference between the maximum and minimum streamwise velocity disturbance over $0<y<\infty$ and $0<z<\pi l$, where $\lambda / 2$ is half from the spanwise separation,

$$
S(x)=\max _{\substack{0<y<\infty \\ 0<z<\lambda / 2}}\left[u(x, y, z)-u_{m}(x, y)\right]-\min _{\substack{0<y<\infty \\ 0<z<\lambda / 2}}\left[u(x, y, z)-u_{m}(x, y)\right]
$$

In figure 7 , we plot this streak amplitude as a function of the streamwise coordinate for all spanwise separations and Mach numbers. It is interesting to notice that there is not a direct relationship with the scaled kinetic energy plotted in the previous figure 6 because the location where the streak amplitude becomes maximum seems to be almost the same for all spanwise separations, which is not the case for the maximum kinetic energy. The level of the streak amplitude corresponding to the smallest spanwise separation of the $M=0.2$ incompressible case is much lower than the other cases; this is a consequence of the weak mixing that was observed in the contour plots in figure $2 \mathrm{a}$. 


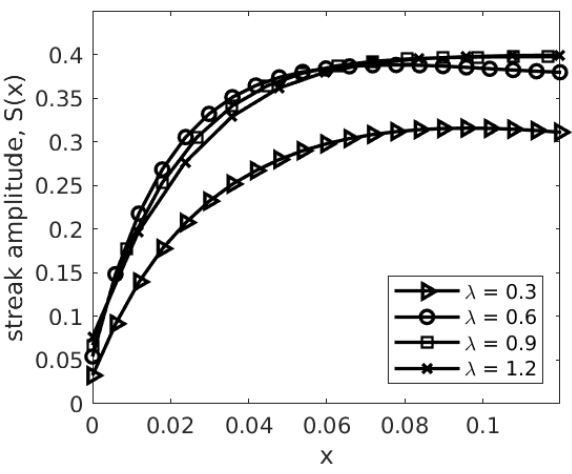

a)

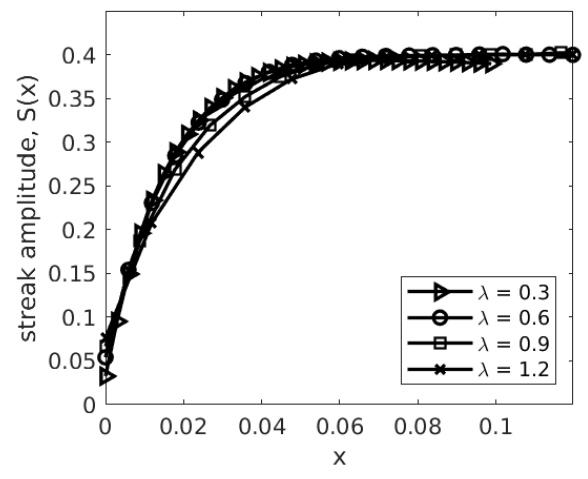

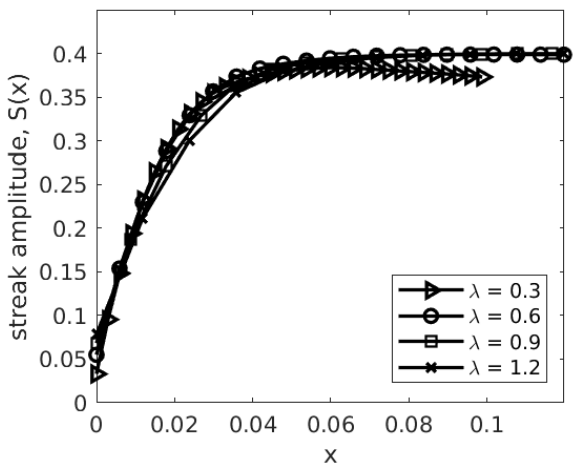

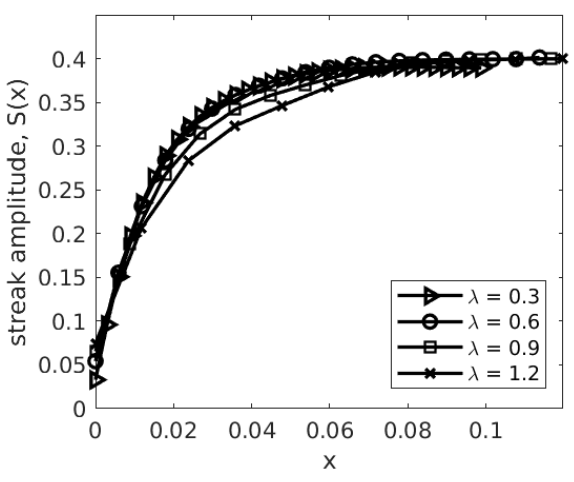

Figure 7. Streak amplitude distribution: a) $M=0.2$; b) $M=0.8$; c) $M=2.0$; d) $M=4.0$.

Finally, we show several typical results from the biglobal linear stability analysis. The growth rates associated with the first and second modes are plotted in figure 8 far all spanwise separations (the Mach number is 0.2 for these results). As expected the maximum growth rates increase as the spanwise separation is increased as was observed from the distribution of kinetic energy. The corresponding wavenumber of the first mode, however, seems to decrease as the spanwise separation is increased, but for the second mode, the wavenumber corresponding to the maximum growth rate increases as the spanwise separation increases.

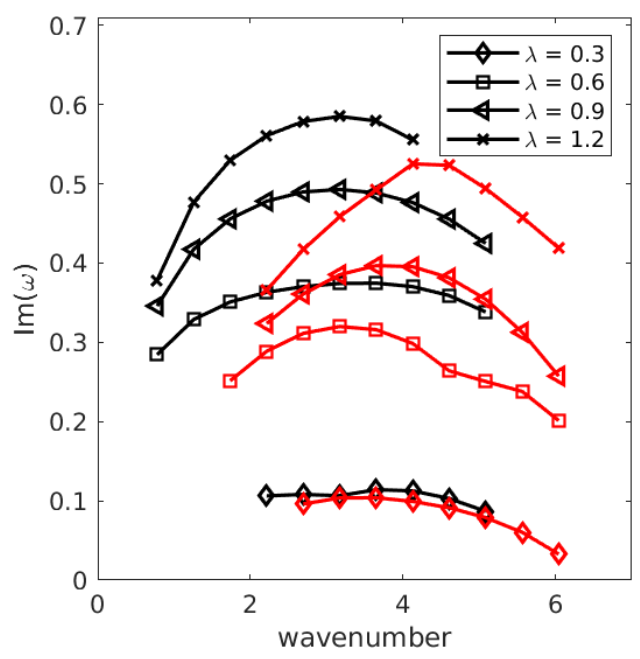

Figure 8. Growth rates. 
Contours of $\hat{u}$-component eigenmodes, corresponding to the first and second highest growth rates, are plotted in figure 9. The eigenmodes are shown in color with the most intense regions highlighted in dark blue, while the black contour lines indicate the base flow. All contour plots suggest that the instability is of varicose type, which is not surprising since the dominant instability in a free shear layer is the KelvinHelmholtz instability which is of varicose type.
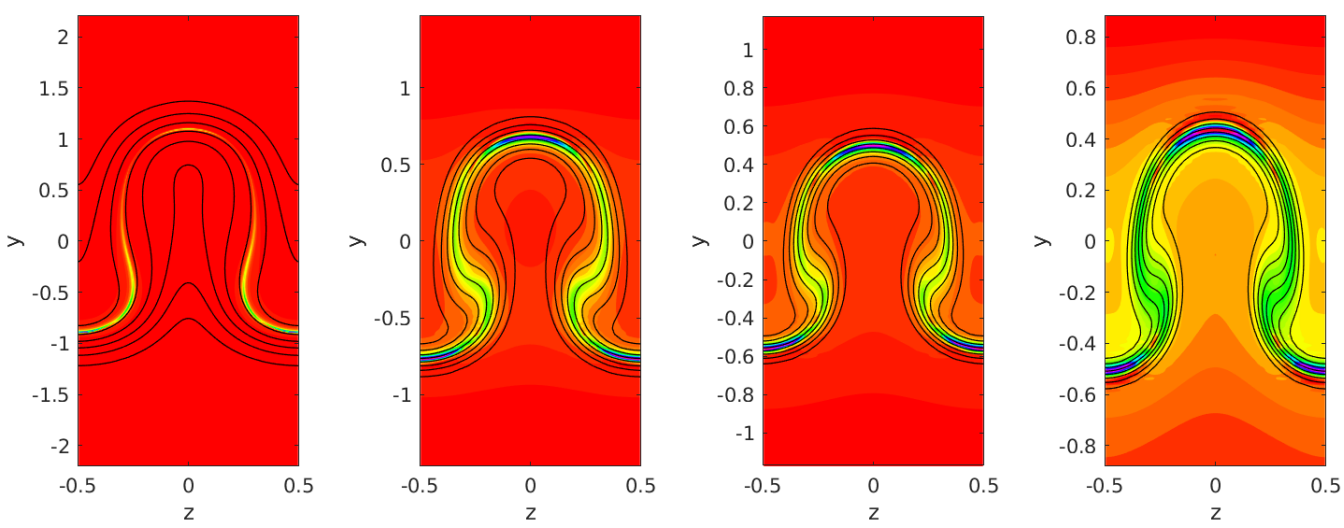

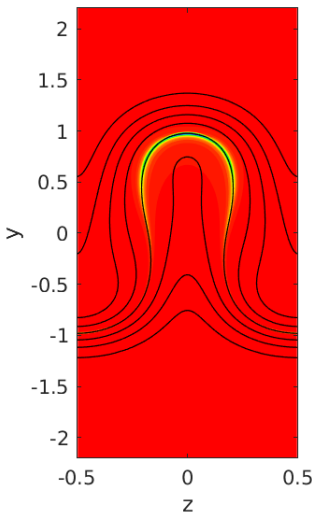

a)

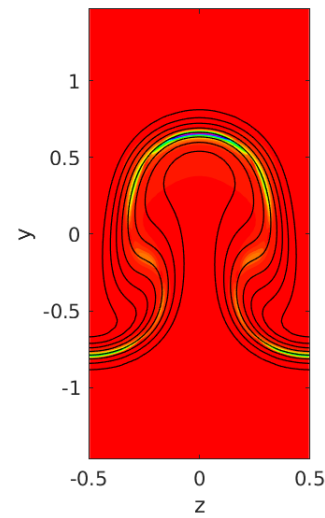

b)

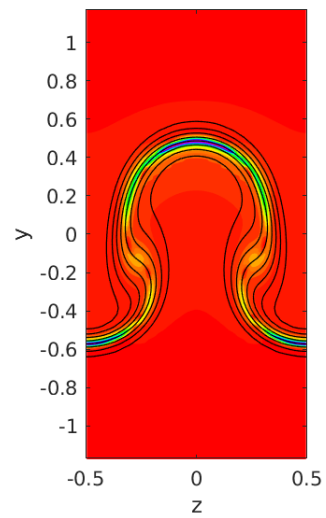

c)

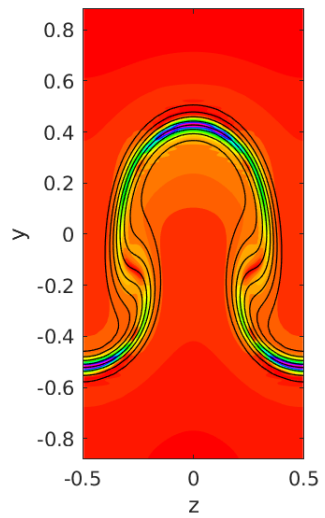

d)

Figure 9. Eigenmodes from the stability analysis (mode 1 in the first row, and mode 2 in the second row): a) $\lambda=0.3$; b) $\lambda=0.6$; b) $\lambda=0.9$; b) $\lambda=1.2$.

\section{Conclusions}

We studied centrifugal instabilities that develop in curved free shear layers in both incompressible and compressible regimes, by using the numerical solution to a parabolized form of the Navier-Stokes equations. We targeted the nonlinear development of these centrifugal instabilities, which resemble many similarities with the Görtler vortices that evolve in boundary layer flows over concave surfaces. The spanwise separation that characterizes the upstream disturbance (and dictate the spanwise separation of the downstream disturbances) and the Mach number of the fastest stream were varied. A biglobal linear stability analysis was also performed to calculate the growth rates of these instabilities, and to determine whether they are of sinuous or varicose type.

A wide range of spanwise separations was considered, and the Mach number covered both the incompressible and compressible regimes. The results showed that for the smallest spanwise separation of $0.3 \mathrm{~cm}$ the centrifugal instability is 'weak' in the sense that the mixing between the two streams is not efficient. As the spanwise separation was increased, the mushroom shapes started to show up, resembling Gortler vortices that are characteristic to boundary layer flows over concave surfaces. The mixing between the two streams increases as the spanwise separation increases, and the large spanwise separations showed spiraling streaks. For the high Mach number cases, the mixing was more efficient, and the spiraling streaks were 
more prevalent at multiple spanwise separations. We also noticed secondary mushroom shapes developing at some spanwise separations. We identified the primary mushroom shape the one that develops as the main instability, and the secondary mushroom shape as the instability that develops from the edge of the main/primary mushroom cap.

The kinetic energy of the instabilities was calculated an plotted against the streamwise coordinate. It was observed that as the spanwise separation is increased, the scaled kinetic energy maximum increases, and that the streamwise location where the energy saturation sets in, moved downstream as the spanwise separation was increased. We also calculated the streak amplitude and plotted it versus the streamwise coordinate, and noticed that the streak amplitude corresponding to the smallest spanwise separation of the $M=0.2$ incompressible case is much lower than the other cases.

From the biglobal linear stability analysis, we plotted the growth rates associated with the first and second modes in figure 8 for all spanwise separations. The maximum growth rates increased as the spanwise separation was increased. The corresponding wavenumber of the first mode, however, seemed to decrease as the spanwise separation was increased, but for the second mode, the wavenumber corresponding to the maximum growth rate increased as the spanwise separation increased. The eigenmodes contour plots suggested that the instability is of varicose type, which is not surprising since the dominant instability in a free shear layer is the Kelvin-Helmholtz instability, which is of varicose type.

\section{Appendix}

The elements of matrices $[A],[B],[C],[D],[E],[F]$, and $[G]$ from the biglobal linear stability analysis are

$$
\begin{gathered}
a_{11}=\bar{W}_{z}-\frac{\bar{T}_{z} \bar{W}}{\bar{T}}+\bar{V}_{y}-\frac{\bar{T}_{y} \bar{V}}{\bar{T}}+i \alpha \bar{U} \\
a_{12}=i \alpha \bar{P} \\
a_{13}=-\frac{\bar{T}_{y} \bar{P}}{\bar{T}}+\bar{P}_{y} \\
a_{14}=-\frac{\bar{T}_{z} \bar{P}}{\bar{T}}+\bar{P}_{z} \\
a_{15}=-\frac{\bar{W}_{z} \bar{P}}{\bar{T}}+\frac{2 \overline{P T}_{z} \bar{W}}{\bar{T}^{2}}-\frac{\bar{P}_{z} \bar{W}}{\bar{T}}-\frac{\bar{V}_{y} \bar{P}}{\bar{T}}+\frac{2 \overline{P T}_{y} \bar{V}}{\bar{T}^{2}}-\frac{\bar{P}_{y} \bar{V}}{\bar{T}}-\frac{i \alpha \overline{P U}}{\bar{T}} \\
a_{21}=\frac{\gamma M^{2} \bar{U}_{z} \bar{W}}{\bar{T}}+\frac{\gamma M^{2} \bar{U}_{y} \bar{V}}{\bar{T}}+i \alpha \\
a_{22}=\frac{i \alpha \gamma M^{2} \overline{P U}}{\bar{T}}+\frac{2 \alpha^{2} \bar{\mu}}{R e}+\frac{\alpha^{2} \bar{\lambda}}{R e} \\
a_{23}=\frac{\gamma M^{2} \overline{P U} \bar{T}_{y}}{\bar{T}}-\frac{i \alpha \bar{\mu}_{\bar{T}} \bar{T}_{y}}{R e} \\
a_{24}=\frac{\gamma M^{2} \overline{P U}_{z}}{\bar{T}}-\frac{i \alpha \bar{\mu}_{\bar{T}} \bar{T}_{z}}{R e} \\
a_{25}=-\frac{\gamma M^{2} \overline{P U}_{z} \bar{W}}{\bar{T}^{2}}-\frac{\gamma M^{2} \overline{P U}_{y} \bar{V}}{\bar{T}^{2}}-\frac{i \alpha \bar{\lambda}_{\bar{T}} \bar{W}_{z}}{R e}-\frac{i \alpha \bar{\lambda}_{\bar{T}} \bar{V}_{y}}{R e}-\frac{\bar{\mu}_{\bar{T}} \bar{U}_{z z}}{R e}-\frac{\bar{\mu}_{\overline{T T}} \bar{T}_{z} \bar{U}_{z}}{R e}-\frac{\bar{\mu}_{\bar{T}} \bar{U}_{y y}}{R e}-\frac{\bar{\mu}_{\overline{T T}} \bar{T}_{y} \bar{U}_{y}}{R e}
\end{gathered}
$$




$$
\begin{aligned}
& a_{31}=\frac{\gamma M^{2} \bar{V}_{z} \bar{W}}{\bar{T}}+\frac{\gamma M^{2} \bar{V}_{y} \bar{V}}{\bar{T}} \\
& a_{32}=-\frac{i \alpha \bar{\lambda}_{\bar{T}} \bar{T}_{y}}{R e} \\
& a_{33}=\frac{\gamma M^{2} \overline{P V}_{y}}{\bar{T}}+\frac{i \alpha \gamma M^{2} \overline{P U}}{\bar{T}}+\frac{\alpha^{2} \bar{\mu}}{R e} \\
& a_{34}=\frac{\gamma M^{2} \overline{P V}_{z}}{\bar{T}} \\
& a_{35}=-\frac{\gamma M^{2} \overline{P V}_{z} \bar{W}}{\bar{T}^{2}}-\frac{\gamma M^{2} \overline{P V}_{y} \bar{V}}{\bar{T}^{2}}-\frac{\bar{\lambda}_{\overline{T T}} \bar{T}_{y} \bar{W}_{z}}{R e}-\frac{\bar{\mu}_{\bar{T}} \bar{W}_{y z}}{R e}-\frac{\bar{\lambda}_{\bar{T}} \bar{W}_{y z}}{R e}-\frac{\bar{\mu}_{\overline{T T}} \bar{T}_{z} \bar{W}_{y}}{R e}-\frac{\bar{\mu}_{\bar{T}} \bar{V}_{z z}}{R e} \\
& -\frac{\bar{\mu}_{\overline{T T}} \bar{T}_{z} \bar{V}_{z}}{R e}-\frac{2 \bar{\mu}_{\bar{T}} \bar{V}_{y y}}{R e}-\frac{\bar{\lambda}_{\bar{T}} \bar{V}_{y y}}{R e}-\frac{2 \bar{\mu}_{\overline{T T}} \bar{T}_{y} \bar{V}_{y}}{R e}-\frac{\bar{\lambda}_{\overline{T T}} \bar{T}_{y} \bar{V}_{y}}{R e}-\frac{i \alpha \bar{\mu}_{\bar{T}} \bar{U}_{y}}{R e} \\
& a_{41}=\frac{\gamma M^{2} \bar{W}_{z} \bar{W}}{\bar{T}}+\frac{\gamma M^{2} \bar{W}_{y} \bar{V}}{\bar{T}} \\
& a_{42}=-\frac{i \alpha \bar{\lambda} \bar{T}_{z}}{R e} \\
& a_{43}=\frac{\gamma M^{2} \overline{P W}_{y}}{\bar{T}} \\
& a_{44}=\frac{\gamma M^{2} \overline{P W}_{z}}{\bar{T}}+\frac{i \alpha \gamma M^{2} \overline{P U}}{\bar{T}}+\frac{\alpha^{2} \bar{\mu}}{R e} \\
& a_{45}=-\frac{\gamma M^{2} \overline{P W}_{z} \bar{W}}{\bar{T}^{2}}-\frac{\gamma M^{2} \overline{P W}_{y} \bar{V}}{\bar{T}^{2}}-\frac{2 \bar{\mu}_{\bar{T}} \bar{W}_{z z}}{R e}-\frac{\bar{\lambda}_{\bar{T}} \bar{W}_{z z}}{R e}-\frac{2 \bar{\mu}_{\overline{T T}} \bar{T}_{z} \bar{W}_{z}}{R e}-\frac{\bar{\lambda}_{\overline{T T}} \bar{T}_{z} \bar{W}_{z}}{R e}-\frac{\bar{\mu}_{\bar{T}} \bar{W}_{y y}}{R e} \\
& -\frac{\bar{\mu}_{\overline{T T}} \bar{T}_{y} \bar{W}_{y}}{R e}-\frac{\bar{\mu}_{T T} \bar{T}_{y} \bar{V}_{z}}{R e}-\frac{\bar{\mu}_{\bar{T}} \bar{V}_{y z}}{R e}-\frac{\bar{\lambda}_{\bar{T}} \bar{V}_{y z}}{R e}-\frac{\bar{\lambda}_{\overline{T T}} \bar{T}_{z} \bar{V}_{y}}{R e}-\frac{i \alpha \bar{\mu}_{\bar{T}} \bar{U}_{z}}{R e} \\
& a_{51}=\frac{\gamma M^{2} \bar{T}_{z} \bar{W}}{\bar{T}}+\frac{\gamma M^{2} \bar{T}_{y} \bar{V}}{\bar{T}}-i \alpha E c \bar{U} \\
& a_{52}=-\frac{2 i \alpha E c \bar{\lambda}_{z}}{R e}-\frac{2 i \alpha E c \bar{\lambda}_{y}}{R e} \\
& a_{53}=\frac{\gamma M^{2} \overline{P T}_{y}}{\bar{T}}-\frac{2 i \alpha E c \bar{\mu} \bar{U}_{y}}{R e}-E c \bar{P}_{y} \\
& a_{54}=\frac{\gamma M^{2} \overline{P T}_{z}}{\bar{T}}-\frac{2 i \alpha E c \bar{\mu} \bar{U}_{z}}{R e}-E c \bar{P}_{z} \\
& a_{55}=-\frac{\gamma M^{2} \overline{P T}_{z} \bar{W}}{\bar{T}^{2}}-\frac{\gamma M^{2} \overline{P T}_{y} \bar{V}}{\bar{T}^{2}}+\frac{i \alpha \gamma M^{2} \overline{P U}}{\bar{T}}-\frac{2 E c \bar{\mu}_{\bar{T}}\left(\bar{W}_{z}\right)^{2}}{R e}-\frac{E c \bar{\lambda}_{\bar{T}}\left(\bar{W}_{z}\right)^{2}}{R e} \\
& -\frac{2 E c \bar{\lambda}_{\bar{T}} \bar{V}_{y} \bar{W}_{z}}{\operatorname{Re}}-\frac{E c \bar{\mu}_{\bar{T}}\left(\bar{W}_{y}\right)^{2}}{R e}-\frac{2 E c \bar{\mu}_{\bar{T}} \bar{V}_{z} \bar{W}_{y}}{R e}-\frac{E c \bar{\mu}_{\bar{T}}\left(\bar{V}_{z}\right)^{2}}{R e}-\frac{2 E c \bar{\mu}_{\bar{T}}\left(\bar{V}_{y}\right)^{2}}{\operatorname{Re}}-\frac{E c \bar{\lambda}_{\bar{T}}\left(\bar{V}_{y}\right)^{2}}{\operatorname{Re}} \\
& -\frac{E c \bar{\mu}_{\bar{T}}\left(\bar{U}_{z}\right)^{2}}{\operatorname{Re}}-\frac{E c \bar{\mu}_{\bar{T}}\left(\bar{U}_{y}\right)^{2}}{\operatorname{Re}}-\frac{\bar{k}_{\bar{T}} \bar{T}_{z z}}{\operatorname{Pr} \operatorname{Re}}-\frac{\bar{k}_{\overline{T T}}\left(\bar{T}_{z}\right)^{2}}{\operatorname{Pr} \operatorname{Re}}-\frac{\bar{k}_{\bar{T}} \bar{T}_{y y}}{\operatorname{Pr} \operatorname{Re}}-\frac{\bar{k}_{\overline{T T}}\left(\bar{T}_{y}\right)^{2}}{\operatorname{Pr} \operatorname{Re}}+\frac{\alpha^{2} \bar{k}}{\operatorname{Pr} \operatorname{Re}} \\
& b_{11}=\bar{V} ; \quad b_{12}=0 ; \quad b_{13}=\bar{P} ; \quad b_{14}=0 ; \quad b_{15}=\frac{\overline{P V}}{\bar{T}} \\
& b_{21}=0 ; \quad b_{22}=\frac{\gamma M^{2} \overline{P V}}{\bar{T}}-\frac{\bar{\mu}_{\bar{T}} \bar{T}_{y}}{R e} ; \quad b_{23}=-\frac{i \alpha \bar{\mu}}{R e}-\frac{i \alpha \bar{\lambda}}{R e} ; \quad b_{24}=0 ; \quad b_{25}=-\frac{\bar{\mu}_{\bar{T}} \bar{U}_{y}}{R e}
\end{aligned}
$$




$$
\begin{aligned}
& b_{31}=1 \\
& b_{32}=-\frac{i \alpha \bar{\mu}}{R e}-\frac{i \alpha \bar{\lambda}}{R e} \\
& b_{33}=\frac{\gamma M^{2} \overline{P V}}{\bar{T}}-\frac{2 \bar{\mu}_{\bar{T}} \bar{T}_{y}}{R e}-\frac{\bar{\lambda}_{\bar{T}} \bar{T}_{y}}{R e} \\
& b_{34}=-\frac{\bar{\mu}_{\bar{T}} \bar{T}_{z}}{R e} \\
& b_{35}=-\frac{\bar{\lambda}_{\bar{T}} \bar{W}_{z}}{R e}-\frac{2 \bar{\mu}_{\bar{T}} \bar{V}_{y}}{R e}-\frac{\bar{\lambda}_{\bar{T}} \bar{V}_{y}}{R e} \\
& b_{41}=0 ; \quad b_{42}=0 ; \quad b_{43}=-\frac{\bar{\lambda}_{\bar{T}} \bar{T}_{z}}{R e} ; \quad b_{44}=\frac{\gamma M^{2} \overline{P V}}{\bar{T}}-\frac{\bar{\mu}_{\bar{T}} \bar{T}_{y}}{R e} ; \quad b_{45}=-\frac{\bar{\mu}_{\bar{T}} \bar{W}_{y}}{R e}-\frac{\bar{\mu}_{\bar{T}} \bar{V}_{z}}{R e} \\
& b_{51}=-E c \bar{V} \\
& b_{52}=-\frac{2 E c \bar{\mu} \bar{U}_{y}}{R e} \\
& b_{53}=-\frac{2 E c \bar{\lambda}_{z}}{R e}-\frac{4 E c \bar{\mu} \bar{V}_{y}}{R e}-\frac{2 E c \bar{\lambda} \bar{V}_{y}}{R e} \\
& b_{54}=-\frac{2 E c \bar{\mu} \bar{W}_{y}}{R e}-\frac{2 E c \bar{\mu} \bar{V}_{z}}{R e} \\
& b_{55}=\frac{\gamma M^{2} \overline{P V}}{\bar{T}}-\frac{2 \bar{k}_{\bar{T}} \bar{T}_{y}}{\operatorname{Pr} R e} \\
& c_{11}=\bar{W} ; \quad c_{12}=0 ; \quad c_{13}=0 ; \quad c_{14}=\bar{P} ; \quad c_{15}=\frac{\overline{P W}}{\bar{T}} \\
& c_{21}=0 ; \quad c_{22}=\frac{\gamma M^{2} \overline{P W}}{\bar{T}}-\frac{\bar{\mu}_{\bar{T}} \bar{T}_{z}}{R e} ; \quad c_{23}=0 ; \quad c_{24}=-\frac{i \alpha \bar{\mu}}{R e}-\frac{i \alpha \bar{\lambda}}{R e} ; \quad c_{25}=-\frac{\bar{\mu}_{\bar{T}} \bar{U}_{z}}{R e} \\
& c_{31}=0 ; \quad c_{32}=0 ; \quad c_{33}=\frac{\gamma M^{2} \overline{P W}}{\bar{T}}-\frac{\bar{\mu}_{\bar{T}} \bar{T}_{z}}{R e} ; \quad c_{34}=-\frac{\bar{\lambda}_{\bar{T}} \bar{T}_{y}}{R e} ; \quad c_{35}=-\frac{\bar{\mu}_{\bar{T}} \bar{W}_{y}}{R e}-\frac{\bar{\mu}_{\bar{T}} \bar{V}_{z}}{R e} \\
& c_{41}=1 \\
& c_{42}=-\frac{i \alpha \bar{\mu}}{R e}-\frac{i \alpha \bar{\lambda}}{R e} \\
& c_{43}=-\frac{\bar{\mu}_{\bar{T}} \bar{T}_{y}}{R e} \\
& c_{44}=\frac{\gamma M^{2} \overline{P W}}{\bar{T}}-\frac{2 \bar{\mu}_{\bar{T}} \bar{T}_{z}}{R e}-\frac{\bar{\lambda}_{\bar{T}} \bar{T}_{z}}{R e} \\
& c_{45}=-\frac{2 \bar{\mu}_{\bar{T}} \bar{W}_{z}}{R e}-\frac{\bar{\lambda}_{\bar{T}} \bar{T}_{z}}{R e}-\frac{\bar{\lambda}_{\bar{T}} \bar{V}_{y}}{R e}
\end{aligned}
$$

16 of 19 


$$
\begin{aligned}
& c_{51}=-E c \bar{W} \\
& c_{52}=-\frac{2 E c \bar{\mu} \bar{U}_{z}}{R e} \\
& c_{53}=-\frac{2 E c \bar{\mu} \bar{W}_{y}}{R e}-\frac{2 E c \bar{\mu} \bar{V}_{z}}{R e} \\
& c_{54}=-\frac{4 E c \bar{\mu} \bar{W}_{z}}{R e}-\frac{2 E c \bar{\lambda} \bar{W}_{z}}{R e}-\frac{2 E c \bar{\lambda}_{y}}{R e} \\
& c_{55}=\frac{\gamma M^{2} \overline{P W}}{\bar{T}}-\frac{2 \bar{k}_{\bar{T}} \bar{T}_{z}}{\operatorname{Pr} \operatorname{Re}} \\
& d_{11}=0 ; \quad d_{12}=0 ; \quad d_{13}=0 ; \quad d_{14}=0 ; \quad d_{15}=0 \\
& d_{21}=0 ; \quad d_{22}=-\frac{\bar{\mu}}{R e} ; \quad d_{23}=0 ; \quad d_{24}=0 ; \quad d_{25}=0 \\
& d_{31}=0 ; \quad d_{32}=0 ; \quad d_{33}=-\frac{2 \bar{\mu}}{R e}-\frac{\bar{\lambda}}{R e} ; \quad d_{34}=0 ; \quad d_{35}=0 \\
& d_{41}=0 ; \quad d_{42}=0 ; \quad d_{43}=0 ; \quad d_{44}=-\frac{\bar{\mu}}{R e} ; \quad d_{45}=0 \\
& d_{51}=0 ; \quad d_{52}=0 ; \quad d_{53}=0 ; \quad d_{54}=0 ; \quad d_{55}=-\frac{\bar{k}}{\operatorname{Pr} R e} \\
& e_{11}=0 ; \quad e_{12}=0 ; \quad e_{13}=0 ; \quad e_{14}=0 ; \quad e_{15}=0 \\
& e_{21}=0 ; \quad e_{22}=0 ; \quad e_{23}=0 ; \quad e_{24}=0 ; \quad e_{25}=0 \\
& e_{31}=0 ; \quad e_{32}=0 ; \quad e_{33}=-\frac{\bar{\mu}}{R e}-\frac{\bar{\lambda}}{R e} ; \quad e_{34}=0 ; \quad e_{35}=0 \\
& e_{41}=0 ; \quad e_{42}=0 ; \quad e_{43}=0 ; \quad e_{44}=-\frac{\bar{\mu}}{R e}-\frac{\bar{\lambda}}{R e} ; \quad e_{45}=0 \\
& e_{51}=0 ; \quad e_{52}=0 ; \quad e_{53}=0 ; \quad e_{54}=0 ; \quad e_{55}=0 \\
& f_{11}=0 ; \quad f_{12}=0 ; \quad f_{13}=0 ; \quad f_{14}=0 ; \quad f_{15}=0 \\
& f_{21}=0 ; \quad f_{22}=-\frac{\bar{\mu}}{R e} ; \quad f_{23}=0 ; \quad f_{24}=0 ; \quad f_{25}=0
\end{aligned}
$$




$$
\begin{gathered}
f_{31}=0 ; \quad f_{32}=0 ; \quad f_{33}=-\frac{\bar{\mu}}{R e} ; \quad f_{34}=0 ; \quad f_{35}=0 \\
f_{41}=0 ; \quad f_{42}=0 ; \quad f_{43}=0 ; \quad f_{44}=-\frac{2 \bar{\mu}}{R e}-\frac{\bar{\lambda}}{R e} ; \quad f_{45}=0 \\
f_{51}=0 ; \quad f_{52}=0 ; \quad f_{53}=0 ; \quad f_{54}=0 ; \quad f_{55}=-\frac{\bar{k}}{\operatorname{Pr} R e} \\
g_{11}=i ; \quad g_{12}=0 ; \quad g_{13}=0 ; \quad g_{14}=0 ; \quad g_{15}=-\frac{i \bar{P}}{\bar{T}} \\
g_{21}=0 ; \quad g_{22}=\frac{i \gamma M^{2} \bar{P}}{\bar{T}} ; \quad g_{23}=0 ; \quad g_{24}=0 ; \quad g_{25}=0 \\
g_{31}=0 ; \quad g_{32}=0 ; \quad g_{33}=\frac{i \gamma M^{2} \bar{P}}{\bar{T}} ; \quad g_{34}=0 ; \quad g_{35}=0 \\
g_{51}=0 ; \quad g_{42}=0 ; \quad g_{43}=0 ; \quad g_{44}=\frac{i \gamma M^{2} \bar{P}}{\bar{T}} ; \quad g_{45}=0 \\
g_{52}=0 ; \quad g_{53}=0 ; \quad g_{54}=0 ; \quad g_{55}=\frac{i \gamma M^{2} \bar{P}}{\bar{T}}
\end{gathered}
$$

\section{References}

${ }^{1}$ Bell, J. H. \& Mehta, R. D. (1990) Development of a two-stream mixing layer within tripped and untripped boundary layers, AIAA J., Vol. 28, pp. 2034-2042.

${ }^{2}$ Boiko, A.V., Ivanov, A.V., Kachanov, Y.S. and Mischenko, D.A. (2010) Steady and unsteady Görtler boundary-layer instability on concave wall, European Journal of Mechanics B/Fluids, Vol. 29, pp. 61-83.

${ }^{3}$ Day, H.P., Herbert, T. and Saric, W.S. (1990) Comparing local and marching analyses of Görtler instability, AIAA Journal, Vol. 28, pp. 1010-1015.

${ }^{4}$ Depmsey, L.D., Hall, P. \& Deguchiu, K. (2017) The excitation of Gortler vortices by free stream coherent structures, J. Fluid Mech., Vol. 826, pp. 60-96.

${ }^{5}$ N. Gregory, N. and Walker, W.S. (1956) The effect on transition of isolated surface excrescences in the boundary-layer 1ARC Technical Report 13, pp. 436.

${ }^{6}$ Goldstein, M., Sescu, A., Duck, P. and Choudhari, M. (2010) The Long Range Persistence of Wakes behind a Row of Roughness Elements, Journal of Fluid Mechanics, Vol. 644, pp. 123-163.

${ }^{7}$ Görtler, H . (1941) Instabilita-umt laminarer Grenzchichten an Konkaven Wanden gegenber gewissen dreidimensionalen Storungen, ZAMM, Vol. 21, pp. 250-52; english version: NACA Report 1375 (1954)

${ }^{8}$ Hall, P. (1982) Taylor-Görtler vortices in fully developed or boundary-layer flows: linear theory, Journal of Fluid Mechanics, Vol. 124, pp. 475-494.

${ }^{9}$ Hall, P. (1983) The linear development of Görtler vortices in growing boundary layers, Journal of Fluid Mechanics, Vol. 130, pp. 41-58.

${ }^{10}$ Hall, P. and Horseman. N. (1991) The linear inviscid secondary instability of longitudinal vortex structures in boundary layers. Journal of Fluid Mechanics. Vol. 232, pp. 357-375.

${ }^{11} \mathrm{Hu}$, F. Q., Otto, S. R. \& Jackson, T. L. (1994) On the stability of a curved mixing layer. In Proc. ICASE workshop on Transition, Turbulence and Combustion (ed. M. Y. Hussaini, T. B. Gatski \& T. L. Jackson), pp. 107-116. Kluwer.

${ }^{12} \mathrm{Li}$, F. and Malik, M. (1995) Fundamental and subharmonic secondary instabilities of Görtler vortices, Journal of Fluid Mechanics, Vol. 297, pp. 77-100.

${ }^{13}$ Li, F., Choudhari, M., Chang, C.-L., Greene, P., and Wu, M. (2010) Development and Breakdown of Gortler Vortices in High Speed Boundary Layers", 48th AIAA Aerospace Sciences Meeting Including the New Horizons Forum and Aerospace Exposition, Aerospace Sciences Meetings. 
${ }^{14}$ Liou, W. W. (1994) Linear instability of curved free shear layers, Phys. Fluids, Vol. 6, pp. 541-549.

${ }^{15}$ Liu. X., Osher, S. and Chan, T. (1994) Weighted essentially non-oscillatory schemes, Journal of Computational Physics, Vol. 115, pp. 200-212.

${ }^{16}$ Malik, M.R. \& Hussaini, M.Y. (1990) Numerical simulation of interactions between Gortler vortices and TollmienSchlichting waves. J. Fluid Mech., Vol. 210, pp. 183-199.

${ }^{17}$ A. Michalke, A. (1965), On spatially growing disturbances in an inviscid shear layer, J. Fluid Mech., Vol. 23, pp. 521.

${ }^{18}$ Otto, S. R., Jackson, T. L. \& Hu, F. Q. 1996 On the spatial evolution of centrifugal instabilities within curved incompressible mixing layers, J. Fluid Mech., Vol. 315, pp. 85-103.

${ }^{19}$ Plesniak, M. W., Mehta, R. D. \& Johnson, J. P. (1994) Curved two-stream turbulent mixing layers: three-dimensional structure and streamwise evolution, J. Fluid Mech., Vol. 270, pp. 1-50.

${ }^{20}$ Plesniak, M. W., Mehta, R. D. \& Johnson, J. P. (1996) Curved two-stream turbulent mixing layers revisited, Expl Therm. Fluid Sci., Vol. 13, pp. 190-205.

${ }^{21}$ Lord Rayleigh (1880) On the stability, or instability of certain fluid motions, Proc. London Math. Soc., Vol. 11 , pp. 57.

${ }^{22}$ Ren, J. \& Fu, S. (2017) Secondary instabilities of Gortler vortices in high-speed boundary layer flows, J. Fluid Mech., Vol. 781, pp. 388-421.

${ }^{23}$ Saric, W.S. (1994) Görtler Vortices, Annu. Rev. Fluid Mech., Vol. 26, pp. 379-409.

${ }^{24}$ Sescu, A., Taoudi, L. and Afsar, M. (2017) Iterative control of Görtler vortices via local wall deformations, Theoretical and Computational Fluids Dynamics, DOI 10.1007/s00162-017-0440-2.

${ }^{25}$ Sescu, A., Pendyala, R. and Thompson, D. (2014) On the Growth of Görtler Vortices Excited by Distributed Roughness Elements, AIAA Paper 2014-2885.

${ }^{26} \mathrm{Sescu}, \mathrm{A}$. and Thompson, D. (2015) On the Excitation of Görtler Vortices by Distributed Roughness Elements, Theoretical and Computational Fluids Dynamics, Vol. 29, pp. 67-92.

${ }^{27}$ Squire, H.B (1933) On the stability of three-dimensional disturbances of viscous flow between parallel walls, Proc. $R$. Soc. London Ser. A, Vol. 142, pp. 621.

${ }^{28}$ Swearingen, J.D. and Blackwelder, R.F. (1987) The growth and breakdown of streamwise vortices in the presence of a wall. J. Fluid Mech., Vol. 182, pp. 255-290.

${ }^{29}$ Tam, C.K.W. and Webb, J.C. (1993), Dispersion-relation-preserving finite difference schemes for Computational Aeroacoustics, Journal of Computational Physics, Vol. 107, pp. 262-281.

${ }^{30}$ Tani, I. (1962) Production of longitudinal vortices in the boundary-layer along a curved wall, J. Geophys. Res., Vol. 67, pp. 3075 .

${ }^{31} \mathrm{Wu}, \mathrm{X}$, Zhao, D. and Luo, J (2011) Excitation of steady and unsteady Görtler vortices by free-stream vortical disturbances, Journal of Fluid Mechanics, Vol. 682, pp. 66-100.

${ }^{32}$ Xu, D., Zhang, Y. \& Wu, X. (2017) Nonlinear evolution and secondary instability of steady and unsteady Gortler vortices induced by free-stream vortical disturbances, J. Fluid Mech., Vol. 829, pp. 681-730. 